\title{
Ю.И. Дорогов
}

\section{УСТОЙЧИВОСТЬ ГОРИЗОНТАЛЬНОГО УПРУГОГО СТЕРЖНЯ}

Исследуется устойчивость горизонтального стержня, лежащего на абсолютно жестком основании и находящегося под действием силы тяжести. Установлено значение критической силы, при котором становится возможной смена прямолинейной формы равновесия на изогнутую. Это значение превосходит значение силы, вычисленное по формуле Эйлера, и зависит от плотности материала стержня. Исследован частичный изгиб стержня в процессе потери устойчивости и найдены условия, при которых такой изгиб становится возможным.

Ключевые слова: устойчивость горизонтального стержня; влияние силь тяжести на критическую силу; частичный изгиб.

\section{1. Устойчивость прямолинейной формы равновесия горизонтального стержня}

При исследовании потери устойчивости стержня, как правило, предполагается, что стержень вертикальный и невесомый [1-3]. В [4] рассмотрена задача об устойчивости вертикального стержня, находящегося под действием собственного веса. Устойчивость сжатого стержня при наличии поперечной нагрузки рассматривалась в [1-3]. В $[1,2,5]$ исследовалась устойчивость сжатого стержня на линейноупругом основании или в упругой среде, когда его изгибу препятствует распределенная поперечная нагрузка, пропорциональная величине прогибов. В работах [6-14] исследовалось влияние разрушения опор, особенностей приложения нагрузки и особенностей опирания на процесс потери устойчивости. Потеря устойчивости стержня при наличии препятствий изгибу исследовалась в [15-17]. Влияние силы тяжести на критическую силу - в [17-19].

В данной работе исследуется устойчивость упругого прямолинейного горизонтального стержня, сжимаемого силой $P$, лежащего на абсолютно жесткой плоской поверхности, не допускающей смещения точек стержня вниз, но не ограничивающей их перемещение вверх (рис. 1).

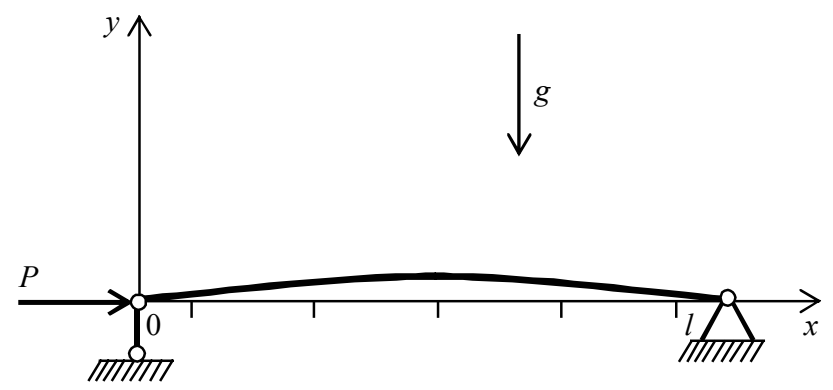

Рис. 1. Горизонтальный упругий стержень на жёстком основании

Fig. 1. Horizontal elastic bar on a rigid base 
Стержень постоянного поперечного сечения с моментом инерции $J$ и площадью поперечного сечения $S$ имеет длину $L$. Его концы шарнирно закреплены. Один конец неподвижен, а другой смещается по мере укорочения стержня и последующего изгиба. Расстояние между концами стержня обозначено $l$. На стержень действует сила тяжести, распределенная равномерно с интенсивностью $q=\rho g S$ по его длине, где $\rho$ - плотность материала стержня; $g$ - ускорение свободного падения. Действие опор на стержень определяется сосредоточенными силами реакции, а действие горизонтальной опорной плоскости непрерывно распределено по поверхности контакта. Задача является статически неопределимой, реакции опор и жесткой поверхности не могут быть найдены из условий равновесия.

При отсутствии опорной плоскости прямолинейная форма равновесия стержня невозможна. Уже в исходном состоянии при нулевом значении сжимающей силы стержень имеет начальную погибь, обусловленную своим весом.

Опорная плоскость препятствует прогибу стержня под действием силы тяжести и, в частности, исключает начальную погибь. Стержень может изгибаться только вверх, против действия силы тяжести. Если при отсутствии опорной плоскости сила тяжести увеличивает изгиб стержня, делая изначально прямолинейную форму равновесия невозможной, то при наличии опорной плоскости сила тяжести направлена против прогиба и способствует устойчивости прямолинейной формы равновесия.

В процессе сжатия и последующего изгиба стержня его подвижный конец перемещается в направлении действия сжимающей силы. Это перемещение складывается из абсолютной деформации стержня на этапе сжатия, пока форма равновесия остается прямолинейной, и смещения конца изогнутого стержня, приближенно равного разности его длины и проекции на первоначальную ось

$$
\Delta=\varepsilon L+L-l .
$$

Здесь $\varepsilon=P / E S$ - относительная деформация сжатия стержня, $E$ - модуль упругости материала стержня.

Принята система координат с центром в подвижном шарнире. Ось $O x$ направлена вдоль прямолинейной оси стержня в сторону его неподвижного конца, ось $O y$ направлена вертикально вверх - в сторону смещения точек оси после потери устойчивости. На начальном этапе изгиба, когда прогибы незначительны, можно принять, что кривизна изогнутой оси стержня равна второй производной функции прогиба $y(x)$. Уравнение равновесия моментов преобразуется к неоднородному линейному дифференциальному уравнению четвертого порядка с постоянными коэффициентами:

$$
y^{\prime \prime \prime \prime}+\alpha^{2} y^{\prime \prime}=-\beta ; \alpha^{2}=P / E J ; \beta=\rho g S / E J .
$$

В соответствии с условиями закрепления, граничные условия запишутся в виде

$$
y(0)=y(l)=0, y^{\prime \prime}(0)=y^{\prime \prime}(l)=0 .
$$

Дифференциальное уравнение (1.2) соответствует прогибам сжимаемого стержня, находящегося под действием силы тяжести как в случае наличия плоской поверхности, препятствующей прогибам стержня вниз, так и в случае отсутствия такой поверхности. Среди решений данного уравнения, удовлетворяющих граничным условиям (1.3), имеются положительные решения, соответствующие изгибу стержня выпуклостью вверх, и отрицательные решения, соответствующие естественному изгибу стержня вниз при отсутствии опорной плоскости. 
Общее решение неоднородного линейного дифференциального уравнения четвертого порядка (1.2) имеет вид

$$
y=-\frac{\beta x^{2}}{2 \alpha^{2}}+C_{1} x+C_{2}+C_{3} \cos \alpha x+C_{4} \sin \alpha x .
$$

Подставляя общее решение (1.4) в граничные условия (1.3) и выражая неопределенные коэффициенты, получим систему уравнений

$$
\left.\begin{array}{l}
C_{1}=\frac{\beta l}{2 \alpha^{2}}, C_{2}=-C_{3}=\beta / \alpha^{4}, \\
2 \sin \frac{\alpha l}{2}\left(\frac{\beta}{\alpha^{4}} \sin \frac{\alpha l}{2}+C_{4} \cos \frac{\alpha l}{2}\right)=0 .
\end{array}\right\}
$$

Последнее уравнение системы имеет два решения. В первом случае $\alpha l \neq 2 \pi$ и $C_{4}=-\beta \alpha^{-4} \operatorname{tg}(\alpha l / 2)$. Коэффициент $C_{4}$ остается неопределенным, так как неопределена длина проекции стержня $l$. Решение краевой задачи запишется в виде

$$
y=\frac{\beta}{2 \alpha^{2}} x(l-x)+\frac{\beta}{\alpha^{4}}(1-\cos \alpha x)+C_{4} \sin \alpha x
$$

Физическому смыслу данной задачи удовлетворяет только положительное решение. Отрицательное соответствует изгибу стержня под действием сжимающей силы и силы тяжести в случае отсутствия опорной поверхности. Если $\alpha l<\pi$, peшение будет отрицательным. Положительное решение становится возможным при $\alpha l>\pi$. Для $\alpha l=\pi$ формула (1.5) дает бесконечные прогибы, за счет последнего неограниченного слагаемого. Следовательно, значение $\alpha l=\pi$ соответствует наименьшему значению нагрузки, при котором становится возможной изогнутая форма равновесия горизонтального стержня, лежащего на жесткой поверхности. Это значение может быть найдено по классической формуле Эйлера для длины $l$ :

$$
P_{l}=\pi^{2} E J / l^{2} .
$$

Второй случай, когда $\alpha l=2 \pi$ и коэффициент $C_{4}$ также оказывается неопределенным, соответствует значительно большей продольной нагрузке и выходит за рамки данного исследования.

Решение (1.5) не может являться уравнением изогнутой оси стержня, так как, с одной стороны, допускает бесконечные прогибы при значениях $\alpha l \rightarrow \pi+0$, a с другой - содержит неопределенную величину $l$.

Бесконечность прогибов является следствием неточности линеаризованного дифференциального уравнения, при составлении которого кривизна заменялась второй производной функции прогиба и, кроме того, допускалось неограниченное увеличение длины изогнутого стержня. На самом деле, изменение длины стержня незначительно по сравнению с самой длиной.

Полученный результат позволяет сделать следующее заключение: 1) изогнутая форма равновесия стержня, лежащего на жесткой горизонтальной опоре, невозможна при значениях сжимающей силы, меньших, чем соответствующее значение силы Эйлера $\left.P_{e}=\pi^{2} E J / L^{2} ; 2\right)$ неограниченность последнего слагаемого в выражении (1.5), при значениях $\alpha l \rightarrow \pi+0$, указывает на то, что остальные слагаемые 
не играют существенной роли по сравнению с последним. Поэтому выражение (1.5) может быть заменено приближенным равенством

$$
y=C \sin \frac{\pi x}{l} .
$$

Здесь $C$ - неопределенный и неограниченный при $\alpha l \rightarrow \pi$ коэффициент.

Для определения коэффициента $C$ будем считать, что длина оси стержня не изменяется, то есть из всех возможных кривых, удовлетворяющих уравнению (1.7), выбираем ту, которая удовлетворяет дополнительному изопериметрическому условию, выражающему постоянство длины стержня

$$
L=\int_{0}^{l} \sqrt{1+y^{\prime 2}} d x .
$$

Здесь $y^{\prime}$ - тангенс угла наклона касательной к оси стержня.

Как и при выводе дифференциального уравнения, ограничимся рассмотрением только тех кривых изгиба стержня, для которых $y^{\prime}(x)<<1$. Тогда условие (1.8), с учетом разложения подынтегрального выражения в ряд Маклорена с двумя первыми членами, можно заменить приближенным равенством

$$
L \approx \int_{0}^{l}\left[1+\frac{y^{\prime 2}}{2}\right] d x .
$$

Подставим первую производную функции (1.7) в подынтегральное выражение и интегрируем его. Из полученного уравнения выразим зависимость коэффициента $C$ от длины проекции $l$ :

$$
C=\frac{2}{\pi} \sqrt{l(L-l)} .
$$

Для невесомого стержня сила Эйлера представляет собой нижнюю границу значений сжимающей силы, при которых становятся возможными изогнутые формы равновесия. При этом прямолинейная форма равновесия перестает быть устойчивой и любое, сколь угодно малое, возмущение вынуждает стержень изменить прямолинейную форму равновесия на устойчивую криволинейную форму равновесия. Таким образом, для невесомого стержня сила, хоть немного превосходящая силу Эйлера, не только может удерживать его в изогнутом состоянии, но и принуждает стержень изогнуться и сменить неустойчивую прямолинейную форму равновесия на устойчивую криволинейную форму равновесия. Для весомого горизонтального стержня, лежащего на жестком основании, это не верно. Сжимающая сила, лишь незначительно превосходящая силу Эйлера, способна удерживать стержень в соответствующем изогнутом состоянии, но не может принудить его к такому состоянию. Прямолинейная форма равновесия остается устойчивой. Действительно, пусть ось стержня приобрела некоторое случайное отклонение $y(x)$ от горизонтальной прямой и пусть функция, выражающая это отклонение, раскладывается в ряд Фурье по синусам

$$
y=\sum_{k=1}^{\infty} b_{k} \sin \frac{\pi k x}{l} .
$$

Здесь коэффициенты Фурье $b_{k}$ положительны, так как $y(x) \geq 0$. 
Стержень будет устойчивым, если сумма моментов внешних сил и внутренних усилий в каждом сечении будет направлена таким образом, чтобы стремиться вернуть стержень в исходное невозмущенное состояние. То есть стержень устойчив, если

$$
P y-0.5 \rho g S x(l-x)+E J y^{\prime \prime}<0 .
$$

Подставляя (1.10) в последнее неравенство, преобразуем его к виду

$$
\sum_{k=1}^{\infty}\left(P-k^{2} P_{l}\right) b_{k} \sin \frac{\pi k x}{l}-0.5 \rho g S x(l-x)<0 .
$$

Неравенство (1.11) будет выполняться при некоторых достаточно малых положительных значениях $P-P_{l}$.

Таким образом, при сколь угодно малом отклонении, внешние силы стремятся вернуть стержень в исходное состояние. Сила $P$, незначительно превосходящая силу Эйлера, не способна изогнуть стержень, и, следовательно, потери устойчивости прямолинейной формы равновесия не происходит. Сила Эйлера не является нижней границей значений сжимающей силы, при которых стержень может принять изогнутое состояние равновесия.

\section{2. Критическая сила}

Линейная плотность энергии сжато-изогнутого силой $P_{l}$ стержня

$$
\tau=\frac{P_{l}^{2}}{2 E S}+\frac{E J \kappa^{2}}{2} .
$$

Здесь $к-$ кривизна изогнутой оси стержня. Приняв $\kappa=y^{\prime \prime}$, учитывая (1.7), (1.9) и интегрируя $\tau$ по длине стержня, получим упругую потенциальную энергию, накопленную в стержне в процессе сжатия и изгиба

$$
W=\frac{P_{l}^{2} L}{2 E S}+P_{l}(L-l)
$$

Изменение гравитационной потенциальной энергии стержня, обусловленное его подъемом над опорной поверхностью вследствие изгиба, равно

$$
\Pi=\rho g S \int_{0}^{l} y d x .
$$

Подставляя в подынтегральное выражение функцию (1.7), с учетом формулы (1.9), после интегрирования получим

$$
\Pi=\frac{4 \rho g S}{\pi^{2}} l^{3 / 2} \sqrt{L-l} .
$$

Полная механическая энергия, накопленная стержнем в изогнутом состоянии, равна $W+\Pi$.

Работа силы $P_{l}$ на перемещении $\Delta$, определяемом формулой (1.1), равна

$$
A=\frac{P_{l}^{2} L}{2 E S}+P_{l}(L-l) .
$$


Сравнивая полученную работу с полной механической энергией, накопленной стержнем, приходим к неравенству $A<W+П$. Таким образом, работы силы $P_{l}$ недостаточно для того, чтобы стержень смог изогнуться и преодолеть силу тяжести. Работа сжимающей силы $P_{l}$ полностью исчерпывается упругой энергией, накапливаемой в стержне $A=W$. В случае невесомого или вертикального стержня, когда энергия не расходуется на преодоление силы тяжести, сила $P_{l}$, удерживающая стержень в изогнутом состоянии, является также и силой, приводящей его к этому состоянию. В рассматриваемом случае, силы, способной удержать стержень в изогнутом состоянии равновесия, оказывается недостаточно, чтобы привести его к такому состоянию.

Для того чтобы консервативная система перешла из одного равновесного состояния в другое, необходимо, чтобы разность потенциальных энергий системы в этих состояниях равнялась работе внешних сил, приложенных к системе.

Рассмотрим жесткое нагружение стержня, при котором задается перемещение конца стержня, а величина сжимающей нагрузки определяется в соответствии с этим перемещением. Такое нагружение может быть реализовано в статически неопределимой системе, элементом которой является исследуемый стержень. Предположим, что стержень сохраняет прямолинейную форму равновесия вплоть до нагрузки $P>P_{l}$, соответствующей перемещению $\Delta$, после чего теряет устойчивость и изгибается. До тех пор пока он сжимается, сохраняя прямолинейную форму равновесия, нагрузка растет пропорционально абсолютной деформации

$$
P=E S \Delta / L \text {. }
$$

После потери устойчивости и изгиба, стержень примет изогнутую форму, соответствующую значению сжимающей силы, меньшему, чем то, которое было достигнуто в процессе сжатия. При выбранном способе нагружения, в процессе изгиба сжимающая сила уменьшится. Концы стержня при этом повернутся, оставаясь неподвижными. Вследствие уменьшения сжимающей силы, деформация сжатия стержня также пропорционально уменьшится. Перемещение $\Delta$ его подвижного конца определяется формулой (1.1), в которой $\varepsilon=P_{l} / E S$ - оставшаяся относительная деформация сжатия стержня после его выпучивания.

Подставляя $\Delta$ в формулу (2.1), получим

$$
P=P_{l}+E S(L-l) / L \text {. }
$$

Продольная сосредоточенная сила совершает работу только на стадии сжатия стержня, так как при изгибе концы стержня неподвижны. Эта работа равна

$$
A=P \Delta / 2 \text {. }
$$

В последнюю формулу подставим выражения для $P$ и $\Delta$. После выполнения элементарных преобразований запишем

$$
A=\frac{P_{l}^{2} L}{2 E S}+P_{l}(L-l)+\frac{E S}{2 L}(L-l)^{2} .
$$

Приравнивая работу сжимающей силы к полной механической энергии, накапливаемой в стержне, получим уравнение

$$
A=W+\Pi \text {. }
$$


Учитывая, что на начальном этапе изгиба длина горизонтальной проекции стержня незначительно отличается от длины стержня, преобразуем последнее равенство к виду

$$
L-l=4\left(\frac{\rho g}{\pi^{2} E}\right)^{2 / 3} L^{5 / 3} .
$$

Подставляя полученное выражение в (2.2) и учитывая, что в начале процесса изгиба $P_{l} \approx P_{e}$, получим

$$
P_{*}=\frac{\pi^{2} E J}{L^{2}}+4 S E^{1 / 3}\left(\frac{\rho g L}{\pi^{2}}\right)^{2 / 3} .
$$

Значение силы, определяемое выражением (2.3), является критическим значением сжимающей силы, под действием которой горизонтальный стержень, лежащий на жесткой поверхности, начнет изгибаться. Критическая сила представляет собой сумму силы Эйлера и компоненты, определяемой влиянием веса. Эта вторая компонента увеличивается вместе с длиной стержня. Поэтому критическая сила увеличивается с увеличением длины, а не уменьшается, как это следовало из формулы Эйлера. Функция $P_{*}(L)$, выражающая зависимость критической силы от длины стержня, имеет минимум при

$$
L_{\min }=\left(\left(\frac{\sqrt{3}}{2}\right)^{3} \frac{\pi^{5} c^{2} i^{3}}{g}\right)^{1 / 4} .
$$

Здесь $i=\sqrt{J / S}-$ радиус инерции сечения стержня, $c=\sqrt{E / \rho}-$ скорость распространения продольных волн в материале стержня. Так, например, для стали $L_{\min }=150,77 i^{3 / 4}$.

Минимальное значение критической силы

$$
P_{\min }=4\left(\frac{4}{3}\right)^{3 / 4} \rho c S \sqrt{\frac{g i}{\pi}} .
$$

При этом второе слагаемое в (2.3), обусловленное учетом силы тяжести, составляет 3/4 от найденного значения, то есть в три раза превышает силу, найденную по формуле Эйлера.

\section{3. Частичный изгиб стержня}

При потере устойчивости вертикальный стержень изгибается сразу по всей длине. Это также верно и для невесомого горизонтального стержня. Для длинного тяжелого горизонтального стержня, лежащего на абсолютно жесткой поверхности, в процессе потери устойчивости становится возможным частичный изгиб. Изгибается только часть стержня, в то время как другая его часть остается прямолинейной и горизонтальной (рис. 2). В соответствии с этим граничные условия должны быть записаны не для концов стержня, а для концов его изогнутой части. Обозначим длину изогнутой части стержня $\Lambda$, а длину проекции изогнутой части на плоскость опоры $\lambda$. 
Полагая, что изогнутая часть начинается у одного из концов стержня, поместим начало системы координат в этом конце и направим ось абсцисс горизонтально в сторону другого конца стержня, а ось ординат вертикально вверх.

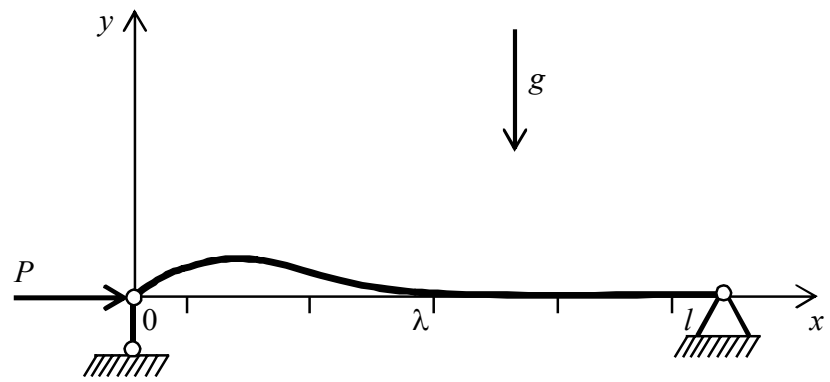

Рис. 2. Изгиб части стержня

Fig. 2. Partial bend of the bar

Функция прогибов оси изогнутой части удовлетворяет дифференциальному уравнению (1.2).

Граничные условия на конце стержня соответствуют условиям шарнирного закрепления. Граничные условия на границе изогнутой и прямолинейной частей стержня являются условиями непрерывности и гладкости изогнутой оси. При указанном выборе системы координат, граничные условия запишутся в виде

$$
y(0)=y(\lambda)=0, y^{\prime}(\lambda)=0, y^{\prime \prime}(0)=0 .
$$

Общее решение неоднородного линейного дифференциального уравнения четвертого порядка (1.2) имеет вид (1.4).

Физическому смыслу рассматриваемой задачи удовлетворяет положительно определенное решение, которое становится возможным при значениях $\alpha \lambda>v$. Величина $v=4,49341$ является корнем уравнения

$$
v=\operatorname{tg} v \text {. }
$$

Таким образом, изогнутая форма равновесия стержня, лежащего на жесткой горизонтальной опоре, становится возможной при значении сжимающей силы

$$
P_{\lambda}=v^{2} E J / \lambda^{2} \text {. }
$$

При значениях $\alpha \lambda<v$ граничным условиям (3.1) удовлетворяют только отрицательные решения, которые соответствуют прогибам стержня длины $\lambda$ с одним шарнирным и одним заделанным концами, сжимаемого продольной силой $P$ и находящегося под действием силы тяжести, при отсутствии опорной плоскости.

И положительное, и отрицательное решения становятся неограниченными при значениях $\alpha \lambda \rightarrow v$.

Пренебрегая конечными слагаемыми в положительном решении, как не играющими существенной роли в сравнении с бесконечным слагаемым, получим приближенное уравнение, описывающее конфигурацию изогнутой оси стержня

$$
y=C(\sin \alpha x-\alpha x \cos \alpha \lambda) .
$$

Здесь $C$ - неограниченный при $\alpha \lambda \rightarrow v$ коэффициент, определяемый из граничных условий (3.1). Этот коэффициент, вообще говоря, является неопределенным, так как неопределены величины $\alpha$ и $\lambda$. Выразим коэффициент $C$ через величи- 
ну $\lambda$, используя изопериметрическое условие, которое в данном случае запишется в виде

$$
\int_{0}^{\lambda} \sqrt{1+y^{\prime 2}} d x=\Lambda
$$

Заменим корень в подынтегральном выражении рядом Маклорена с двумя первыми членами и подставим в полученное равенство производную выражения (3.3). После интегрирования и преобразования, для определения $C$ получим уравнение

$$
4(\Lambda-\lambda) \lambda=C^{2} v^{2} \sin ^{2} v .
$$

Учитывая уравнение (3.2) и приближенное равенство $\alpha \lambda=v$, из последнего равенства выразим зависимость коэффициента $C$ от длины проекции $\lambda$ :

$$
C=k \sqrt{\lambda(\Lambda-\lambda)} ; k=2 v^{-2} \sqrt{1+v^{2}} \approx 0,45599 .
$$

Упругая энергия, накопленная стержнем в процессе сжатия и частичного изгиба, равна

$$
W=\frac{P_{\lambda}^{2} L}{2 E S}+P_{\lambda}(\Lambda-\lambda)
$$

Изменение потенциальной гравитационной энергии стержня при $\alpha \lambda \rightarrow v+0$ может быть преобразовано к виду

$$
\Pi=\varsigma \rho g S \lambda^{3 / 2} \sqrt{\Lambda-\lambda} ; \varsigma=\left(\sqrt{1+v^{2}}+1\right)^{2} / v^{3} .
$$

При жестком нагружении перемещение подвижного конца стержня

$$
\Delta=\varepsilon L+\Lambda-\lambda .
$$

Здесь $\varepsilon=P_{\lambda} / E S$ - относительная деформация сжатия стержня, оставшаяся после изгиба его части.

Сжимающая сила, необходимая для того, чтобы абсолютная деформация стержня достигла величины (3.5),

$$
P=P_{\lambda}+E S(\Lambda-\lambda) / L .
$$

Работа силы $P$ на перемещении $\Delta$

$$
A=\frac{P \Delta}{2}=\frac{P_{\lambda}^{2} L}{2 E S}+P_{\lambda}(\Lambda-\lambda)+\frac{E S}{2 L}(\Lambda-\lambda)^{2} .
$$

Приравняем найденную работу к полной механической энергии, накопленной стержнем. Приводя в полученном равенстве подобные слагаемые и учитывая, что для начального момента выпучивания $\lambda \approx \Lambda$, после упрощения получим

$$
\Lambda-\lambda=\xi\left(\frac{\rho g L}{E}\right)^{2 / 3} \Lambda ; \xi=2^{2 / 3} v^{-2}\left(\sqrt{1+v^{2}}+1\right)^{4 / 3} \approx 0,78246 .
$$

Подставляя разность $\Lambda-\lambda$, найденную по формуле (3.7), в выражение (3.6) получим

$$
P=\xi\left(\frac{(\rho g)^{2} E}{L}\right)^{1 / 3} S \Lambda+\frac{v^{2} E J}{\Lambda^{2}} .
$$


На рис. 3 изображен схематический график зависимости $P(\Lambda)$. Из этого графика видно, что зависимость $P(\Lambda)$ достигает минимума $P_{*}$ при некотором значении длины изогнутой части $\Lambda_{*}$. При $P=P_{*}$ уравнение (3.8) имеет единственное решение $\Lambda=\Lambda_{*}$, которое может быть найдено как корень уравнения

$$
P^{\prime}(\Lambda)=0 \text {. }
$$

Разрешая последнее уравнение и выполняя преобразования, найдем корень

$$
\Lambda_{*}=\zeta\left(\frac{c^{4} i^{6} L}{g^{2}}\right)^{1 / 9} ; \zeta=\left(2 v^{2} / \xi\right)^{1 / 3} \approx 3,72312 .
$$

Если $\Lambda_{*}<L$, стержень в процессе потери устойчивости начинает изгибаться не по всей длине, а только по части, имеющей длину $\Lambda_{*}$. Если $\Lambda_{*}>L$, стержень изгибается сразу по всей своей длине.

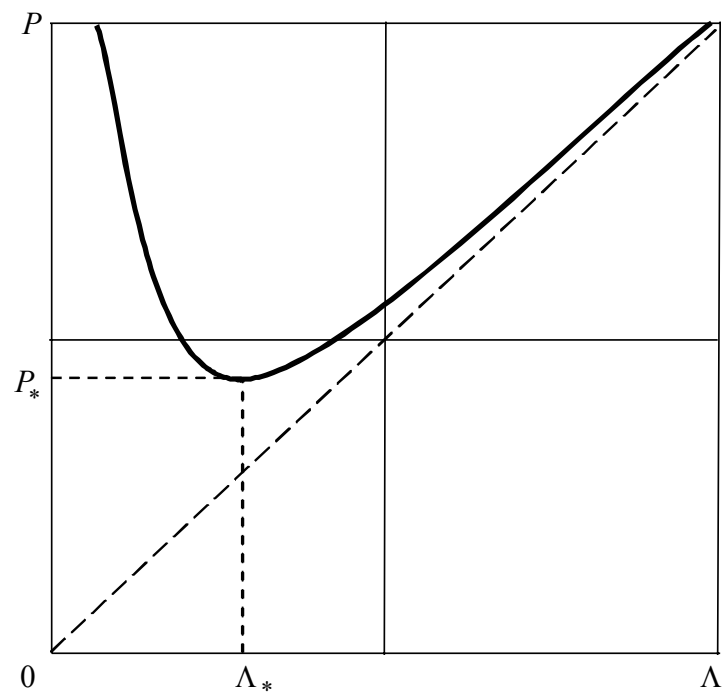

Рис. 3. График зависимости

Fig. 3. Dependency diagram

Подставляя выражение $\Lambda_{*}$ в (3.8) и преобразовывая полученное выражение, найдем

$$
P_{*}=3 \psi \rho S c^{10 / 9}\left(\frac{g^{2} i^{3}}{L}\right)^{2 / 9} ; \psi=(\nu \xi / 2)^{2 / 3} \approx 1,45659 .
$$

При $P<P_{*}$ уравнение (3.8) не имеет решений, и, следовательно, стержень сохраняет устойчивость прямолинейной формы равновесия.

При $P>P_{*}$ уравнение (3.8) имеет два решения. Меньшее решение не соответствует физическому смыслу задачи. В противном случае пришлось бы принять, что с увеличением сжимающей силы длина изогнутой части стержня уменьшает- 
ся, а это противоречит наблюдаемому явлению. Больший корень уравнения (3.8) является значением длины изогнутой части стержня при заданном значении сжимающей силы $P>P_{*}$.

Таким образом, $P_{*}$ является минимальным значением сжимающей силы, при котором горизонтальный стержень, лежащий на жестком основании, начинает изгибаться. Другими словами, $P_{*}$ является критическим значением, при котором начинается процесс потери устойчивости горизонтального стержня, лежащего на жестком основании. При этом стержень изгибается не полностью, а только частью длины $\Lambda_{*}$.

По мере увеличения нагрузки, при $P>P_{*}$, длина $\Lambda$ изогнутой части стержня увеличивается в соответствии с формулой (3.8), пока не станет равной $L$. Однако формула (3.8) является справедливой лишь на начальной стадии изгиба, при малых значениях угловых коэффициентов наклона изогнутой оси стержня $y^{\prime}$.

Такому частичному изгибу подвержены достаточно длинные стержни. Равенство $\Lambda_{*}=L$ позволяет определить наименьшую длину стержня, при которой частичный изгиб возможен

$$
L_{0}=\zeta^{9 / 8}\left(\frac{c^{2} i^{3}}{g}\right)^{1 / 4} .
$$

Так, например, для стали $L_{0} \approx 176,2 i^{3 / 4}$.

Если длина стержня меньше величины $L_{0}$, то стержень при потере устойчивости будет изгибаться по всей длине. Значение критической силы, при котором начнется процесс потери устойчивости, определяется формулой (2.3).

Более длинные стержни при потере устойчивости начнут изгибаться только по части своей длины. По мере увеличения нагрузки изогнутая часть стержня удлиняется, пока изгиб не охватит весь стержень.

\section{4. Выводы}

1. При сжатии тяжелого горизонтального стержня, лежащего на жестком основании, сила Эйлера не является критической силой, при которой происходит потеря устойчивости прямолинейной формы равновесия. Критическая сила значительно превосходит силу Эйлера и зависит от плотности материала стержня и его деформационно-прочностных свойств.

2. С увеличением плотности материала критическая сила увеличивается. Поэтому для более плотных и менее упругих материалов учет силы тяжести, при расчете горизонтального стержня на устойчивость, является более актуальным.

3. Критическая сила зависит от длины стержня, и эта зависимость имеет минимум для некоторого значения длины $L_{\min }$. Минимальное значение критической силы в 4 раза превосходит силу Эйлера для данной длины стержня. Для более длинных стержней, критическая сила увеличивается с увеличением длины.

4. Достаточно длинные стержни, для которых $L>L_{0}$, при потере устойчивости начинают изгибаться не по всей длине, а лишь по ее части. По мере увеличения нагрузки изогнутая часть стержня увеличивается до тех пор, пока не охватит всю длину. 


\section{ЛИТЕРАТУРА}

1. Тимошенко С.П. Устойчивость стержней, пластин и оболочек. М.: Наука, 1971.

2. Вольмир А.С. Устойчивость деформируемых систем. М.: Наука, 1967.

3. Лейтес С.Д. Устойчивость сжатых стальных стержней. М.: Государственное издательство по строительству и архитектуре, 1954.

4. Пановко Я.Г., Губанова И.И. Устойчивость и колебания упругих систем. М.: Наука, 1987.

5. Работнов Ю.Н. Механика деформируемого твердого тела. М.: Наука, 1988.

6. Дорогов Ю.И. О потере устойчивости абсолютно жесткого стержня с разрушающейся опорой // Механика композиционных материалов и конструкций. 2006. № 3. С. 300-311.

7. Дорогов Ю.И. Устойчивость упругого стержня с разрушающейся опорой // Механика композиционных материалов и конструкций. 2010. № 1. С. 84-96.

8. Дорогов Ю.И. Продольный изгиб стержня с разрушающимися заделками // Механика композиционных материалов и конструкций. 2010. № 4. С. 575-586.

9. Дорогов Ю.И. Влияние жёсткости и прочности опоры на продольный изгиб стержня // Вестник Северного (Арктического) федерального университета. Естественные науки. 2015. № 3. С. 108-116.

10. Дорогов Ю.И. Об устойчивости формы незакреплённого упругого стержня с жёсткими полками на концах // Прикладная математика и механика. 2013. Т. 77. Вып. 3. С. 462-473.

11. Дорогов Ю.И. Устойчивость стержня с искривленными торцами // Механика композиционных материалов и конструкций. 2012. № 2. С. 255-266.

12. Дорогов Ю.И. Устойчивость стержня с жёсткими окончаниями // Строительная механика и расчёт сооружений. 2013. № 3. С.16-21.

13. Дорогов Ю.И. Об одном случае потери устойчивости стержня под действием следящей силы // Строительство и реконструкция. 2016. № 1 (63). С. 3-9.

14. Дорогов Ю.И. Устойчивость стержня, вдавливаемого в жёсткий шпур с упругим дном // Строительная механика и расчёт сооружений. 2015. № 3. С. 34-41.

15. Дорогов Ю.И. Применение изопериметрического условия в задачах потери устойчивости стержня // Вестник Томского государственного университета. Математика и механика. 2014. № 4 (30). С. 71-81.

16. Дорогов Ю.И. Устойчивость стержня при наличии препятствий выпучиванию // Вестник Томского государственного университета: Математика и механика. 2015. № 4(36). C. $71-84$.

17. Дорогов Ю.И. Потеря устойчивости горизонтального стержня с грузом и упругой опорой посредине // Строительная механика и расчет сооружений. 2014. № 2. С. 31-39.

18. Дорогов Ю.И. Об устойчивости горизонтального стержня, лежащего на жёстком основании // Строительная механика и расчет сооружений. 2015. № 1. С. 58-65.

19. Дорогов Ю.И. Моделирование потери устойчивости тяжёлого стержня на жёстком основании // Вестник ЮУрГУ. Серия «Математика. Механика. Физика». 2016. Т. 8. № 1. C. $34-42$.

Статья поступила 30.04.16 г.

Dorogov Yu.I. (2016) STABILITY OF A HORIZONTAL ELASTIC BAR. Tomsk State University Journal of Mathematics and Mechanics. 4(42). pp. 70-83

DOI $10.17223 / 19988621 / 42 / 7$

Stability of a horizontal bar lying on an absolutely rigid base in the gravity force is investigated. The base prevents the bar from deflection by the force of gravity and, in particular, excludes the initial deflection. The bar can be bent only upward, against the gravity force. In the absence of the supporting plane, the gravity force increases the bend of the bar, which makes the initial rectilinear form of balance impossible; in the presence of the supporting plane, the gravity force is directed against the deflection and promotes the stability of the rectilinear form of balance. 
The possibility of the curved bar balance forms adjacent to a rectilinear form is considered.

It is shown that Euler's force is not the lower bound value of the compressing force, sufficient for transformation of the bar from a rectilinear form of balance to a curved form of balance. The value of a critical force which makes such transition possible is obtained. The critical force significantly exceeds the corresponding value of the force calculated by Euler's formula and depends not only on the stress-related properties of bar material but also on its density. The critical force is determined from the condition of the equality of the compressing force work and total potential energy of the curved bar, including the elastic energy and gravitational energy.

The bend of the bar in process of stability loss in some part of the bar length, while the other part remains rectilinear and horizontal, is investigated. Conditions under which such bend becomes possible are found. It is shown that the length of the curved part of the bar increases with an increase in the compressing force.

Keywords: stability of the horizontal bar, effect of gravity force on the critical force, partial bend.

DOROGOV Yury Ivanovich (Candidate of Technical Sciences,

Branch of Moscow Power Engineering Institute (National Research University)

in Volzhskiy, Volzhskiy, Russian Federation)

E-mail: ydorogov@yandex.ru

\section{REFERENCES}

1. Timoshenko S.P. (1971) Ustoychivost' sterzhney, plastin i obolochek [Stability of bars, plates, and shells]. Moscow: Nauka.

2. Vol'mir A.S. (1967) Ustoychivost' deformiruemykh sistem [Stability of deformable systems]. Moscow: Nauka.

3. Leytes S.D. (1954) Ustoychivost' szhatykh stal'nykh sterzhney [Stability of compressed steel bars]. Moscow: Gosudarstvennoe izdatel'stvo po stroitel'stvu i arkhitekture [State publishing on construction and architecture].

4. Panovko Ya.G., Gubanova I.I. (1987) Ustoychivost' i kolebaniya uprugikh sistem [Stability and oscillations of elastic systems] Moscow: Nauka.

5. Rabotnov Yu.N. (1988) Mekhanika deformiruemogo tverdogo tela [Mechanics of deformable solids]. Moscow: Nauka.

6. Dorogov Yu.I. (2006) O potere ustoychivosti absolyutno zhestkogo sterzhnya s razrushayushcheysya oporoy [Stability loss of a rigid bar with crumbling support]. Mekhanika kompozitsionnykh materialov i konstruktsiy - Composite Mechanics and Design. 3. pp. 300-311.

7. Dorogov Yu.I. (2010) Ustoychivost' uprugogo sterzhnya s razrushayushcheysya oporoy [Stability of an elastic bar with crumbling support]. Mekhanika kompozitsionnykh materialov $i$ konstruktsiy - Composite Mechanics and Design. 1. pp. 84-96.

8. Dorogov Yu.I. (2010) Prodol'nyy izgib sterzhnya s razrushayushchimisya zadelkami [Longitudinal bending of the rod with crumbling attachment]. Mekhanika kompozitsionnykh materialov $i$ konstruktsiy - Composite Mechanics and Design. 4. pp. 575-586.

9. Dorogov Yu.I. (2015) Vliyanie zhestkosti i prochnosti opory na prodol'nyy izgib sterzhnya [Effect of the support tensile strength and rigidity on longitudinal bend of the rod]. Vestnik Severnogo (Arkticheskogo) federal'nogo universiteta. Estestvennye nauki - Northern (Arctic) Federal University Journal. Natural Science. 3. pp. 108-116. DOI 10.17238/issn22276572.2015.3.108.

10. Dorogov Yu.I. (2013) The stability of the shape of an unattached elastic rod with stiff flanges on its ends. Journal of Applied Mathematics and Mechanics. 3. pp. 338-345.

11. Dorogov Yu.I. (2012) Ustoychivost' sterzhnya s iskrivlennymi tortsami [Stability of the rod with distorted butts]. Mekhanika kompozitsionnykh materialov i konstruktsiy - Composite Mechanics and Design. 2. pp. 255-266.

12. Dorogov Yu.I. (2013) Ustoychivost' sterzhnya s zhestkimi okonchaniiami [Stability of the rod with rigid butts]. Stroitel'naia mekhanika i raschet sooruzhenii - Structural Mechanics and Analysis of Constructions. 3. pp.16-21. 
13. Dorogov Yu.I. (2016) Ob odnom sluchae poteri ustoychivosti sterzhnya pod deystviem sledyashchey sily [About one case of rod stability loss on effect of the following force]. Stroitel'stvo i rekonstruktsiya - Building and reconstruction. 1(63). pp. 3-9.

14. Dorogov Yu.I. (2015) Ustoychivost' sterzhnya, vdavlivaemogo v zhestkiy shpur s uprugim dnom [Stability of the column pressed into the rigid blast hole with the elastic bottom]. Stroitel'naya mekhanika i raschet sooruzheniy - Structural Mechanics and Analysis of Constructions]. 3. pp. 34-41.

15. Dorogov Yu.I. (2014) Primenenie izoperimetricheskogo usloviya v zadachakh poteri ustoychivosti sterzhnya [Application of the isoperimetric condition in problems of buckling for a rod]. Vestnik Tomskogo gosudarstvennogo universiteta. Matematika i mekhanika - Tomsk State University Journal of Mathematics and Mechanics. 4(30). pp. 71-81.

16. Dorogov Yu.I. (2015) Ustoychivost' sterzhnya pri nalichii prepyatstviy vypuchivaniyu [Stability of a column in the presence of obstacles to buckling]. Vestnik Tomskogo gosudarstvennogo universiteta: Matematika i mekhanika [Tomsk State University Journal of Mathematics and Mechanics]. 4 (36). pp. 71-84. DOI 10.17223/19988621/36/9.

17. Dorogov Yu.I. (2014) Poterya ustoychivosti gorizontal'nogo sterzhnya s gruzom i uprugoy oporoy posredine [Stability loss of the horizontal rod with load and elastic support in the middle]. Stroitel'naya mekhanika i raschet sooruzheniy - Structural Mechanics and Analysis of Constructions. 2. pp. 31-39.

18. Dorogov Yu.I. (2015) Ob ustoychivosti gorizontal'nogo sterzhnya, lezhashchego na zhestkom osnovanii [Stability of the horizontal rod, lying on the rigid base]. Stroitel'naya mekhanika $i$ raschet sooruzheniy - Structural Mechanics and Analysis of Constructions]. 1. pp. 58-65.

19. Dorogov Yu.I. (2016) Modelirovanie poteri ustoychivosti tyazhelogo sterzhnya na zhestkom osnovanii [The modeling of loss in stability of a heavy rod placed on a rigid basis]. Vestnik YuUrGU. Seriya «Matematika. Mekhanika. Fizika»-Bulletin of the South Ural State University Series "Mathematics. Mechanics. Physics". 8(1). pp. 34-42. DOI 10.14529/ mmph160105. 